\title{
Imperfect Fermi gas: Kinetic and interaction energies
}

\author{
A. A. Shanenko* \\ TFVS, Universiteit Antwerpen, Universiteitsplein 1, B2610 Antwerpen, Belgium
}

(Received 12 March 2004; published 21 December 2004)

\begin{abstract}
A uniform ground-state three-dimensional Fermi gas with short-range repulsive pairwise interaction is under consideration. Its kinetic and interaction energies are calculated up to the second order of the expansion in the gas parameter. Similar to recent results for an interacting Bose gas, the quantities in question are found to depend on the pairwise interaction through two characteristic lengths: the former, $a$, is the $s$-wave scattering length, and the latter, $b$, is related to $a$ by $b=a-m(\partial a / \partial m)$, where $m$ stands for the fermion mass. To control the results, we proceed in two independent ways. The first involves the Hellmann-Feynman theorem applied to derive the kinetic and interaction energies from the total-energy expansion in the gas parameter first found by Huang and Yang. The second way operates with in-medium pair wave functions and allows one to calculate the quantities of interest "from scratch." The results of the present investigation, taken together with those of the recent consideration of a dilute Bose gas, make it possible to conclude that the pairwise interaction in a quantum gas has an essential and nontrivial effect on the kinetic energy, which is not the case for a classical many-particle system.
\end{abstract}

DOI: 10.1103/PhysRevA.70.063618

PACS number(s): 03.75.Ss, 05.30.Fk, 05.70.Ce

\section{INTRODUCTION}

Recent experiments with magnetically trapped alkali atoms have significantly renewed interest in the properties of quantum gases. As is known, the initial series of these experiments concerned a Bose gas $\left({ }^{87} \mathrm{Rb}[1],{ }^{23} \mathrm{Na}[2]\right.$, and ${ }^{7} \mathrm{Li}$ [3]), which motivated extensive reconsiderations and some new investigations in the field of Bose-Einstein condensation. The derived theoretical and experimental results not only confirmed conclusions made more than 40 years ago but also extended the understanding of boson physics. In particular, the Gross-Pitaevskii equation developed in the 1960s [4] was found to provide a good agreement with experimental data on the density profiles of a trapped Bose gas [5]. The exact derivation of the Gross-Pitaevskii energy functional [6] can be highlighted among recent theoretical achievements. As for experimental innovations, the interference pattern of two expanding Bose condensates [7,8] is of interest (for theoretical details see the papers Ref. [9] and reviews in $[10,11])$.

The first communications about experiments with trapped fermionic atoms appeared in the literature about five years ago [12] when a temperature near $0.4 T_{f}$ was claimed to be reached for trapped ${ }^{6} \mathrm{Li}$ atoms, where $T_{f}$ is the temperature below which the Fermi statistics is of importance. Today trapped atoms of the fermionic isotope ${ }^{40} \mathrm{~K}$ can be cooled down to temperatures $0.17 T_{f}$ [13] and $0.08 T_{f}$ [14]. So the regime of the degenerate Fermi gas is already under experimental study. In view of this fact, reconsideration of the basic aspects of the theory of an imperfect Fermi gas looks to be of importance.

In the present paper a uniform ground-state threedimensional (3D) Fermi gas with a short-range repulsive

\footnotetext{
*Also at Bogoliubov Laboratory of Theoretical Physics, Joint Institute for Nuclear Research, 141980 Dubna, Russia.
}

pairwise interaction is investigated. Why could the situation of a repulsive Fermi gas be of interest while the $s$-wave scattering length $a$ is negative for ${ }^{6} \mathrm{Li} \mathrm{[15]} \mathrm{and,} \mathrm{most} \mathrm{likely,}$ for ${ }^{40} \mathrm{~K}$ [16]? The point is that the magnetic-field Fesbasch resonances provide experimentalists with a good means of governing the $s$-wave scattering length of the atom-withatom interaction $[13,14,17]$. Indeed, imposing a uniform magnetic field significantly changes this characteristic length in such a way that effectively attractive ( $a$ is negative) and repulsive ( $a$ is positive) regimes are attainable $[13,14,17]$. Thus, experiments with trapped fermionic atoms of ${ }^{6} \mathrm{Li}$ and ${ }^{40} \mathrm{~K}$ offer an exciting possibility to explore an imperfect Fermi gas in both the normal (see, for example, Ref. [17]) and superfluid (see the recent paper in [14]) states.

The particular problem to be investigated here concerns the kinetic $E_{\text {kin }}$ and interaction $E_{\text {int }}$ energies of an interacting Fermi gas. This problem is connected with a more general question as to whether or not the pairwise interaction has an effect on the kinetic energy of a quantum gas. It is well known that for a classical imperfect gas the pairwise potential does not make any contribution to the kinetic energy [18]. As for a quantum gas, common expectations regarding $E_{\text {kin }}$ are based on the pseudopotential approach. This approach suggests that the kinetic energy is not practically sensitive to the pairwise interaction in a quantum gas. To go into detail, the total energy of a ground-state uniform 3D Bose gas, taken in the leading order of the expansion in the gas parameter, coincides with the corresponding interaction energy when calculating with the pseudopotential (see Refs. $[10,19,20])$. For a ground-state uniform 3D Fermi gas with repulsive pairwise interaction the kinetic energy found with the pseudopotential does not include terms coming from the pairwise interaction in both the leading and next-to-leading orders of the expansion in the gas parameter (see Ref. [21] and Eqs. (24) and (25) below). Hence, concerning the influence of the pairwise potential on the kinetic energy, the situation in a quantum gas, from the "pseudopotential" point of view, is the same as in a classical gas. However, recently a 
thorough investigation $[19,20]$ has demonstrated that this is not the case, and the pairwise interaction has a strong and nontrivial effect on the kinetic energy of an interacting Bose gas. The aim of the present paper is to show the same for an imperfect Fermi gas.

The devision of the total energy in the kinetic and interaction energies can seem formal from the "thermodynamic" point of view (when keeping in mind only thermodynamic quantities). However, this division is rather natural. The point is that the kinetic energy is proportional to the second moment of the momentum distribution. In principle, this distribution can be measured directly. On the contrary, the total and interaction energies are only inferred from experimental data by means of model-dependent interpretations. To get information about the momentum distribution of a trapped quantum gas, the so-called time-of-flight expansion images are investigated $[1,2]$. They are used to establish the BoseEinstein condensation and determine the system temperature. Hence, it seems natural to try the same experimental technique in order to find the kinetic energy of a trapped quantum gas. However, some serious problems can be met in this way. In particular, to extract the kinetic energy of the groundstate quantum gas, one needs to remove the contribution of thermal excitations. This is a more complicated problem than observations of the bimodal character of the velocity distribution [2] when establishing the Bose-Einstein condensation. Another point is that to exclude any change in the momentum distribution during the expansion, images corresponding to sufficiently small times of flight should be involved. In this case the resolution problem can prevent us from getting data with a reasonable accuracy. Thus, any measurements of the two-point Green's function can be helpful in the situation of interest.

Note that an important argument in favor of investigations of the kinetic energy of a quantum gas is an interesting interplay between the pairwise interaction and momentum distribution in a quantum many-body system. For example, the present work and papers Refs. $[19,20]$ show that vapors of alkali-metal atoms driven by a magnetic field to the regime of a nearly zero scattering length $[22,23]$ acquire a very unusual feature never met before. Indeed, the total energy in this situation is equal (or practically equal) to that of the corresponding free quantum gas. However, this is not the case for the kinetic and interaction energies: the contributions of pairwise interaction to these quantities are the same (or practically the same) in absolute value but have opposite signs. Other interesting examples of the interplay between the pairwise interaction and kinetic energy can be found in Sec. III and in Ref. [24].

This paper is organized as follows. In Sec. II the kinetic and interaction energies of a ground-state uniform Fermi gas with short-range repulsive pairwise interaction are calculated via the Hellmann-Feynman theorem, starting from the lowdensity expansion for the total energy first found by Huang and Yang [25]. An important point of these calculations is the use of the auxiliary variational theorem for the scattering length proved in Refs. [19,20]. Section III presents a consideration of $E_{\text {kin }}$ and $E_{\text {int }}$ under various regimes: from weak to strong coupling. This consideration is interesting itself but is also needed to discuss the failure of the pseudopotential ap- proach to deal with $E_{\text {kin }}$ and $E_{\text {int }}$. The derivation of the kinetic and interaction energies given in Sec. II is rather formal, which can prevent a reader from understanding the underlying physics in detail. This is why in Sec. IV the leading order of the expansion in the gas parameter for $E_{\mathrm{int}}$ is calculated in a more physically sound way. It is based on a method developed in Refs. [19,20] and operating with inmedium pair wave functions. An advantage of this approach is that it allows for the calculation of $E_{\text {kin }}$ and $E_{\text {int }}$ "from scratch," without using the result of Huang and Yang for the total energy. Section $\mathrm{V}$ shows how the kinetic energy of a Fermi gas can be calculated by means of pair wave functions. Here an important relation between the quantumparticle momentum distribution and in-medium pair wave functions is discussed. Note that exactly this relation is a conductor of the pairwise-interaction influence on the kinetic energy of a quantum gas. In addition, some interesting links to the Bethe-Goldstone equation [26] and Galitskii effective wave functions $[27,28]$ are considered in Sec. V.

\section{HELLMANN-FEYNMAN THEOREM}

Let us consider the system of $N$ identical fermions placed in a box with the volume $V$ and ruled by the Hamiltonian

$$
\hat{H}=-\sum_{i} \frac{\hbar^{2}}{2 m} \nabla_{i}^{2}+\sum_{i>j} V\left(\left|\mathbf{r}_{i}-\mathbf{r}_{j}\right|\right),
$$

with the pairwise interaction $V(r)=\gamma \Phi(r)$, where $\gamma>0$ is the coupling constant and $\Phi(r)>0$ stands for the interaction kernel [29]. The particle spin is assumed to be $s=1 / 2$ [30]. The ground-state energy of the system in question $E=\langle 0|\hat{H}| 0\rangle$ obeys the relation

$$
\delta E=\langle 0|\delta \hat{H}| 0\rangle,
$$

which is often called the Hellmann-Feynman theorem, $\delta E$ and $\delta \hat{H}$ being infinitesimal changes of $E$ and $\hat{H}$ [for example, see the proof of Eq. (2) in Ref. [31]]. An advantage of this theorem is that it yields important relations connecting the total ground-state energy $E$ with the kinetic $E_{\text {kin }}$ and interaction $E_{\text {int }}$ energies. These relations read

$$
\begin{gathered}
-m \frac{\partial E}{\partial m}=\left\langle 0\left|-\sum_{i} \frac{\hbar^{2}}{2 m} \nabla_{i}^{2}\right| 0\right\rangle=E_{\text {kin }}, \\
\gamma \frac{\partial E}{\partial \gamma}=\left\langle 0\left|\sum_{i>j} V\left(\mid \mathbf{r}_{i}-\mathbf{r}_{j}\right)\right| 0\right\rangle=E_{\text {int }} .
\end{gathered}
$$

If the dependence of the ground-state energy on the coupling constant and particle mass were known explicitly, one would readily be able to calculate $E_{\text {kin }}$ and $E_{\text {int }}$ via Eqs. (3) and (4). However, it is not the case as a rule.

In the situation of the repulsive Fermi gas the dependence of the ground-state energy on $\gamma$ and $m$ is indeed known only implicitly. According to the familiar result of Huang and Yang [25] found with the pseudopotential and then reproduced with the boundary collision expansion method [32] and with the approach of the Green's functions [27], the energy per fermion $\varepsilon=E / N$ is of the form 


$$
\varepsilon=\frac{3 \hbar^{2} k_{F}^{2}}{10 m}\left[1+\frac{10}{9 \pi} k_{F} a+\frac{4}{21 \pi^{2}}(11-2 \ln 2) k_{F}^{2} a^{2}\right],
$$

which is accurate to the terms of order $k_{F}^{2} a^{2}$. In Eq. (5), $a$ stands for the $s$-wave scattering length, $k_{F}$ is the Fermi wave number given by

$$
k_{F}=\left(3 \pi^{2} n\right)^{1 / 3}
$$

where $n=N / V$, and the thermodynamic limit $N \rightarrow \infty, V \rightarrow \infty$, $n=N / V \rightarrow$ const is implied. Inserting Eq. (5) in Eqs. (3) and (4), one can arrive at

$$
\begin{gathered}
\varepsilon_{\mathrm{kin}}=\varepsilon-\frac{3 \hbar^{2} k_{F}^{2}}{10 a} \frac{\partial a}{\partial m}\left[\frac{10}{9 \pi} k_{F} a+\frac{8}{21 \pi^{2}}(11-2 \ln 2) k_{F}^{2} a^{2}\right], \\
\varepsilon_{\text {int }}=\frac{3 \hbar^{2} k_{F}^{2}}{10 m a} \gamma \frac{\partial a}{\partial \gamma}\left[\frac{10}{9 \pi} k_{F} a+\frac{8}{21 \pi^{2}}(11-2 \ln 2) k_{F}^{2} a^{2}\right],
\end{gathered}
$$

where $\varepsilon_{\text {kin }}=E_{\text {kin }} / N$ and $\varepsilon_{\text {int }}=E_{\text {int }} / N$. Hence, to derive the kinetic and interaction energies from Eq. (5) with the help of the Hellmann-Feynman theorem, we should have an idea concerning the derivatives of $a$ with respect to the particle mass $m$ and coupling constant $\gamma$. As $E=E_{\text {kin }}+E_{\text {int }}$, then from Eqs. (7) and (8) it follows that

$$
m \partial a / \partial m=\gamma \partial a / \partial \gamma
$$

This property of the derivatives becomes clear if we remind that in the 3D case the $s$-wave scattering length is given by

$$
a=\frac{m \gamma}{4 \pi \hbar^{2}} \int d^{3} r \varphi(r) \Phi(r),
$$

where $\varphi(r)$ obeys the the two-body Schrödinger equation taken in the center-off-mass frame and at the zero energy:

$$
-\left(\hbar^{2} / m \gamma\right) \nabla^{2} \varphi(r)+\Phi(r) \varphi(r)=0 .
$$

The pair wave function $\varphi(r)$ represents the zero-momentum scattering state so that $\varphi(r) \rightarrow 1$ when $r \rightarrow \infty$. The scattering part of the pair wave function given by the definition $\varphi(r)$ $=1+\psi(r)$ is characterized by the asymptotic behavior

$$
\psi(r) \rightarrow-a / r \quad(r \rightarrow \infty) .
$$

As is seen from Eqs. (10) and (11), the scattering length depends on the particle mass and coupling constant through the product $m \gamma$. Hence, to use Eqs. (7) and (8), we should know the derivative of $a$ with respect to $m \gamma$.

This derivative can be found with a variational theorem proved in Refs. [19,20]. After small algebra the result of this theorem can be rewritten as

$$
\delta a=\frac{\delta(m \gamma)}{4 \pi \hbar^{2}} \int d^{3} r \varphi^{2}(r) \Phi(r),
$$

where, recall $\varphi(r)$ is a real quantity. In view of importance of this theorem, let us make some explaining remarks concerning its proof. The key point here is to represent Eq. (10) in the form

$$
a=\frac{m \gamma}{4 \pi \hbar^{2}} \int d^{3} r \varphi^{2}(r) \Phi(r)+\frac{1}{4 \pi} \int d^{3} r|\nabla \psi(r)|^{2},
$$

which follows from Eqs. (11) and (12) and $\nabla(\psi \nabla \psi)$ $=\nabla \psi \nabla \psi+\psi \nabla^{2} \psi$. Varying the left- and right-hand sides of Eq. (14) with respect to $m \gamma$, one can find that $\delta \varphi$ does not make any contribution to $\delta a$ due to Eq. (11). From Eq. (13) one gets

$$
m \partial a / \partial m=\gamma \partial a / \partial \gamma=a-b,
$$

where the additional characteristic length $b>0$ is of the form

$$
b=\frac{1}{4 \pi} \int d^{3} r|\nabla \psi(r)|^{2} .
$$

We emphasize that $b$ is always positive and cannot be represented as a function of $a$ in principle. It means that the ratio $b / a$ depends on a particular shape of the pairwise interaction. Now we have everything at our disposal to calculate the kinetic and interaction energies of the Fermi gas in the ground state. Equations (7) and (8), taken in conjunction with Eq. (15), result in the following expressions:

$$
\begin{gathered}
\varepsilon_{\mathrm{kin}}=\frac{3 \hbar^{2} k_{F}^{2}}{10 m}\left[1+\frac{10}{9 \pi} k_{F} b+\frac{4}{21 \pi^{2}}(11-2 \ln 2)\left(2 \frac{b}{a}\right.\right. \\
\left.-1) k_{F}^{2} a^{2}\right], \\
\varepsilon_{\mathrm{int}}=\frac{3 \hbar^{2} k_{F}^{2}}{10 m}\left(1-\frac{b}{a}\right) \times\left[\frac{10}{9 \pi} k_{F} a+\frac{8}{21 \pi^{2}}(11-2 \ln 2) k_{F}^{2} a^{2}\right],
\end{gathered}
$$

whose sum reproduces Eq. (5). From Eqs. (17) and (18) it is seen that $\varepsilon_{\text {kin }}$ and $\varepsilon_{\text {int }}$ are given by the series expansions in $k_{F} a$ but with the coefficients depending on the ratio $b / a$. The derived results prove that the pairwise interaction influences both the kinetic and interaction energies of a Fermi gas.

\section{FROM WEAK TO STRONG COUPLING}

To go in a more detail concerning Eqs. (17) and (18), let us consider them in various regimes. We speak about weak coupling when the interaction kernel $\Phi(r)$ is integrable and the coupling constant $\gamma \ll 1$. The integrable kernel with $\gamma$ $\gg 1$ and a singular pairwise interaction like the hard-sphere potential are related to the strong-coupling regime. The expansion parameter $k_{F} a$ involved in the expressions mentioned above corresponds to the dilution limit $k_{F} \rightarrow 0$. In this situation one is able to operate with Eq. (5) in the both weakand strong-coupling regimes. However, in the weak-coupling case $k_{F} a$ can be small due to $a \propto \gamma \ll 1$, which makes it possible to rearrange Eq. (5) in such a way that to derive the weak-coupling expansion for $\varepsilon$.

In the weak-coupling regime the scattering length $a$ is given by the Born series:

$$
a=a_{0}+a_{1}+\cdots,
$$

with 


$$
a_{0}=\frac{m \gamma}{4 \pi \hbar^{2}} \Phi(k=0), \quad a_{1}=-\frac{m \gamma^{2}}{4 \pi \hbar^{2}} \int \frac{\mathrm{d}^{3} k}{(2 \pi)^{3}} \frac{\Phi^{2}(k)}{2 T_{k}},
$$

where $T_{k}=\hbar^{2} k^{2} /(2 m)$ and $\Phi(k)$ is the Fourier transform of the interaction kernel (for more detail see Ref. [33]). Inserting Eq. (19) in Eq. (5), one gets

$$
\begin{aligned}
\varepsilon= & \frac{3 \hbar^{2} k_{F}^{2}}{10 m}\left[1+\frac{10}{9 \pi} k_{F} a_{0}+\left(\frac{10}{9 \pi} k_{F} a_{1}+\frac{4}{21 \pi^{2}}\right.\right. \\
& \left.\left.\times(11-2 \ln 2) k_{F}^{2} a_{0}^{2}\right)\right],
\end{aligned}
$$

where the terms of order $\gamma^{3}$ are ignored. Now the dependence of $\varepsilon$ on the particle mass and coupling constant is known explicitly. Therefore, we can readily employ the Hellmann-Feynman theorem that, taken together with Eq. (21), yields

$$
\begin{aligned}
\varepsilon_{\text {kin }}=\frac{3 \hbar^{2} k_{F}^{2}}{10 m} & {\left[1-\left(\frac{10}{9 \pi} k_{F} a_{1}+\frac{4}{21 \pi^{2}}(11-2 \ln 2) k_{F}^{2} a_{0}^{2}\right)\right], } \\
\varepsilon_{\text {int }} & =\frac{3 \hbar^{2} k_{F}^{2}}{10 m}\left[\frac{10}{9 \pi} k_{F} a_{0}+\left(\frac{20}{9 \pi} k_{F} a_{1}+\frac{8}{21 \pi^{2}}(11\right.\right. \\
& \left.\left.-2 \ln 2) k_{F}^{2} a_{0}^{2}\right)\right] .
\end{aligned}
$$

The major part of the $\gamma$-dependent contribution to Eq. (21) is related to $\varepsilon_{\text {int }}$, this part being proportional to $\gamma$, while terms of order $\gamma^{2}$ appear in both $\varepsilon_{\text {kin }}$ and $\varepsilon_{\text {int }}$. This meets the usual expectations that the pairwise potential does not influence practically the kinetic energy of a quantum gas (see, for example, Refs. $[10,15]$ and the discussion in the Introduction of Ref. [19]). However, for $\gamma \gtrsim 1$ the effect of the pairwise interaction on $\varepsilon_{\text {kin }}$ is rather curious and differs significantly from that of the weak-coupling case. But before proceeding to detailed examples, let us consider the pseudopotential predictions for $\varepsilon_{\text {kin }}$ and $\varepsilon_{\text {int }}$ being a part of the usual expectations about the imperfect quantum gases.

The standard way of operating with the thermodynamics of a dilute cold Fermi gas with a repulsive pairwise potential is based on the pseudopotential procedure by Huang and Yang [25]. The key point of this approach is that to go beyond the weak-coupling regime, one should replace the Fourier transform of the pairwise interaction $\Phi(k)$ by the quantity $4 \pi \hbar^{2} a / m$ in all the expressions related to the weakcoupling approximation. In so doing, some singular integrals appear. Indeed, substituting $t=4 \pi \hbar^{2} a / m$ for $\Phi(k)$ in Eq. (14), one gets a divergent quantity $a_{1} \propto \int \mathrm{d}^{3} k / 2 T_{k}$ that makes a contribution to the total energy of the system. To derive the familiar result of Huang and Yang, the divergent term connected with $a_{1}$ should be removed, which is fulfilled via a regularization procedure. In particular, this can be realized by means of replacing $\left(4 \pi \hbar^{2} a / m\right) \delta(r)$ by $\left(4 \pi \hbar^{2} a / m\right) \delta(r)$ $\times(\partial / \partial r) r$. Hence, to generalize Eqs. (21)-(23) to the case of a realistic coupling, one should replace $a_{0}$ by $a$ and remove all the terms depending on $a_{1}$. This yields Eq. (5) and the following pseudopotential predictions for the kinetic and interaction energies:

$$
\begin{gathered}
\varepsilon_{\mathrm{kin}}^{(\mathrm{ps})}=\frac{3 \hbar^{2} k_{F}^{2}}{10 m}\left[1-\frac{4}{21 \pi^{2}}(11-2 \ln 2) k_{F}^{2} a^{2}\right], \\
\varepsilon_{\mathrm{int}}^{(\mathrm{ps})}=\frac{3 \hbar^{2} k_{F}^{2}}{10 m}\left[\frac{10}{9 \pi} k_{F} a+\frac{8}{21 \pi^{2}}(11-2 \ln 2) k_{F}^{2} a^{2}\right] .
\end{gathered}
$$

Note that Eqs. (24) and (25) can be derived in another way, as well. For example, the first term in Eq. (25) is reproduced within the Hartree-Fock approximation used together with the pseudopotential (see Ref. [25] and the next section of the present paper). According to Eqs. (24) and (25) taken to the leading and next-to-leading orders in $k_{F} a$, the pairwise interaction makes a contribution only to the interaction energy, which significantly differs from the correct results given by Eqs. (17) and (18). Thus, the pseudopotential approach has serious limitations due to a problem with the kinetic and interaction energies.

Now let us consider Eqs. (17) and (18) beyond the weakcoupling regime, the ratio $b / a$ being of special interest. We start with the simplified situation of the penetratable spheres that is specified by the interaction kernel

$$
\Phi(r)= \begin{cases}\Phi & \text { if } r \leqslant r_{0}, \\ 0 & \text { if } r>r_{0} .\end{cases}
$$

Inserting Eq. (26) into Eq. (11), one can find

$$
\varphi(r)=\left\{\begin{array}{cl}
2 A \sinh (\alpha r) / r & \text { if } r \leqslant r_{0}, \\
1-a / r & \text { if } r>r_{0},
\end{array}\right.
$$

where $\alpha^{2}=m \gamma \Phi / \hbar^{2}(\Phi>0)$ and $A$ is a constant. Equation (27) taken together with the usual boundary conditions at $r$ $=r_{0}$ leads to

$$
a=r_{0}\left[1-\tanh \left(\alpha r_{0}\right) /\left(\alpha r_{0}\right)\right]
$$

and

$$
b=r_{0}\left[1-\frac{1}{2}\left[3 \tanh \left(\alpha r_{0}\right) /\left(\alpha r_{0}\right)-\operatorname{csch}\left(\alpha r_{0}\right)\right]\right],
$$

where $\operatorname{csch}(x)=1 / \cosh ^{2}(x)$. One can check that in the weakcoupling regime, when $\alpha r_{0} \propto \gamma^{1 / 2} \rightarrow 0$, Eqs. (28) and (29) are reduced to

$$
a \simeq \frac{1}{3} \alpha^{2} r_{0}^{3} \propto \gamma, \quad b \simeq \frac{2}{15} \alpha^{4} r_{0}^{5} \propto \gamma^{2},
$$

and, hence, $b \ll a$. This means that the next-to-leading term in the expansion in $k_{F} a$ given by Eq. (5) is mostly the interaction energy, as was mentioned above. On the contrary, in the strong-coupling regime, when $\alpha r_{0} \rightarrow \infty$, Eqs. (28) and (29) yield

$$
a \rightarrow r_{0}, \quad b \rightarrow r_{0} .
$$

Hence, $b / a \rightarrow 1$ when $\gamma \rightarrow+\infty$, and the ground-state energy of a dilute Fermi gas with the hard-sphere interaction is purely kinetic. Note that the same conclusion is valid for a 
dilute Bose gas of hard spheres $[19,20]$. This result can be understood as follows. Any particle of the system with the hard-sphere pairwise interaction moves in a region bounded by the infinite walls due to the surrounding hard spheres. This is why its energy and, hence, the total system energy are purely kinetic in this case, which was first mentioned by Lieb and Yngvason [34].

A more realistic example concerns a situation when the interaction kernel combines a short-range repulsive sector with a long-range attractive "tail." It is usually considered (see, e.g., [35]) that for alkali atoms one can employ the following approximation:

$$
\Phi(r)=\left\{\begin{array}{cl}
+\infty & \text { if } r \leqslant r_{0}, \\
-C / r^{6} & \text { if } r>r_{0} .
\end{array}\right.
$$

The scattering length for the pairwise-interaction kernel given by Eq. (32) is of the form (see Ref. [36])

$$
a / r_{0}=\sqrt{2 x} \Gamma(3 / 4) J_{-1 / 4}(x) /\left[2 \Gamma(5 / 4) J_{1 / 4}(x)\right],
$$

where $x=r_{c}^{2} /\left(2 r_{0}^{2}\right)$ with $r_{c}=\left(m \gamma C / \hbar^{2}\right)^{1 / 4}$, whereas $J_{\nu}(x)$ and $\Gamma(z)$ denote the Bessel function and the Euler gamma function. We have $J_{\nu}(x) \simeq x^{\nu} /\left[2^{\nu} \Gamma(1+\nu)\right]$ for $x \rightarrow 0$ [37]. Therefore, Eq. (33) reduces to $a / r_{0}=1$ in this limit. In other words, when the attractive sector is "switched off," we arrive at the hard-sphere result discussed in the previous paragraph. For $x>0$ the scattering length (33) is a decreasing function of $x$ with the complicated pattern of behavior specified by the infinite set of singular points $\left\{x_{\infty}^{(1)}, x_{\infty}^{(2)}, x_{\infty}^{(3)}, \ldots\right\}$. These points are the zeros of $J_{1 / 4}(x)$ so that $a \rightarrow-\infty$ when $x \rightarrow x_{\infty}^{(i)}-0$ and $a \rightarrow+\infty$ when $x \rightarrow x_{\infty}^{(i)}+0$. In addition, there is also the infinite sequence of the zeros of the scattering length $\left\{x_{0}^{(1)}, x_{0}^{(2)}, x_{0}^{(3)}, \ldots\right\}$ being the zeros of $J_{-1 / 4}(x)$. Note that $x_{0}^{(i)}<x_{\infty}^{(i)}<x_{0}^{(i+1)}$. Keeping in mind this information, we can turn to the ratio $b / a$ for the pair interaction kernel (32). Equation (33) leads to

$$
\gamma \partial a / \partial \gamma=D \sqrt{x}\left\{J_{-1 / 4}(x) /\left[2 J_{1 / 4}(x)\right]-\sqrt{2} /\left[\pi J_{1 / 4}^{2}(x)\right]\right\},
$$

with $D=r_{0} \Gamma(3 / 4) /\left[2^{3 / 2} \Gamma(5 / 4)\right]$. Note that to derive Eq. (34), the useful formula

$$
J_{\nu+1}(x) J_{-\nu}(x)+J_{\nu}(x) J_{-(\nu+1)}(x)=-2 \sin (\pi \nu) /(\pi x)
$$

should be applied [37]. Equation (34), taken in conjunction with Eq. (15), yields

$$
b / a=3 / 4+1 /\left[\pi \sqrt{2} J_{1 / 4}(x) J_{-1 / 4}(x)\right] .
$$

As is seen from Eq. (35), in the limit $x \rightarrow 0$ we get the hardsphere result $b / a=1$ (see Fig. 1). The quantity $b$ (recall that $b$ is always positive) remains finite at $x=x_{0}^{(i)}$, while $b \rightarrow+\infty$ for $x \rightarrow x_{\infty}^{(i)}$. In the latter situation $b$ goes to infinity in such a way that $b /|a| \rightarrow+\infty$ though $|a| \rightarrow \infty$ for $x \rightarrow x_{\infty}^{(i)}$, too. Hence, $x_{0}^{(i)}$ and $x_{\infty}^{(i)}$ are both singular points of $b / a$. Let us stress that the zeros of the scattering length in the case in question have nothing to do with the weak-coupling regime for which, recall, $b /|a| \ll 1$. Operating with the kernel (32) we are not able to reach the weak-coupling regime at all because this kernel

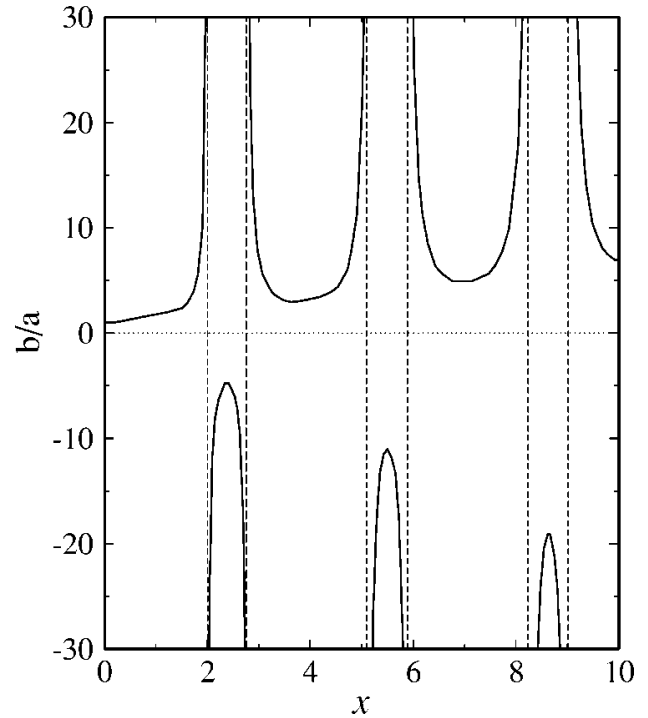

FIG. 1. The ratio $b / a$ versus $x=r_{c}^{2} /\left(2 r_{0}^{2}\right)$ for the pairwise interaction kernel (32), $r_{c}=\left(m \gamma C / \hbar^{2}\right)^{1 / 4}$.

is not bound from above. Now let us consider positive values of the scattering length given by Eq. (33). As seen from Fig. $1, a \geqslant 0$ for $x \leqslant x_{0}^{(1)}$ and $x_{\infty}^{(i)}<x \leqslant x_{0}^{(i)}$. For any of these regions $b / a \geqslant 1$. In particular, $b / a \geqslant 3$ for $x_{\infty}^{(1)}<x \leqslant x_{0}^{(1)}$ whereas $b / a \geqslant 5$ for $x_{\infty}^{(2)}<x \leqslant x_{0}^{(2)}$. Hence, the interaction energy for the pairwise potential specified by Eq. (32) is negative even for the positive values of the scattering length $(C>0$ is meant). Moreover, at $r_{c} / r_{0} \gg 1$, when the role of the attractive "tail" is significant, we have $b / a \gg 1$. In this situation the contribution of the pairwise interaction to the kinetic energy is much larger than its contribution to the mean energy. Hence, the interaction energy is negative in this case and also much larger, if taken in absolute value, than the sum of the $a$-dependent terms in the Huang-Yang result. In view of the recent consideration of a trapped Fermi gas [17], features of the ratio $b / a$ near the special points $x_{\infty}^{(i)}$ and $x_{0}^{(i)}$ can be of particular interest. Imposing a uniform magnetic field, experimentalists can change the scattering length in such a way that $a$ approaches the Fesbasch-resonance point $(a \rightarrow \infty)$ or drops down to zero $[14,17]$. In the both situations, as follows from Eqs. (17) and (18), we have $b /|a| \gg 1$. Note that passage to the limit $a \rightarrow \infty$ in Eqs. (17) and (18) is not correct because it violates the expansion condition $k_{F}|a| \ll 1$. On the contrary, one can put $a=0$, which leads to

$$
\begin{gathered}
\varepsilon_{\mathrm{kin}}(a \rightarrow 0)=\frac{3 \hbar^{2} k_{F}^{2}}{10 m}+\frac{\hbar^{2} k_{F}^{3} b}{3 \pi m}, \\
\varepsilon_{\mathrm{int}}(a \rightarrow 0)=-\frac{\hbar^{2} k_{F}^{3} b}{3 \pi m} .
\end{gathered}
$$

Equations (36) and (37) correspond to an interesting and extreme situation: the total energy is the same as for an ideal Fermi gas, while the interaction and kinetic energies taken separately have nothing to do with the case of noninteracting fermions. 
Thus, the examples given above show that the correct results for the kinetic and interaction energies given by Eqs. (17) and (18) differ significantly from the pseudopotential predictions (24) and (25). Contrary to a classical imperfect gas, the pairwise interaction in the Fermi gas has a profound effect on its kinetic energy, which well meets the conclusions for an interacting Bose gas in Refs. $[19,20]$. To go into more detail about this feature of the quantum gases and display the underlying physics, the interaction and kinetic energies of the uniform ground-state 3D Fermi gas with repulsive forces are investigated with the help of the in-medium pair wave functions in Secs. IV and V.

\section{INTERACTION ENERGY VIA THE PAIR WAVE FUNCTIONS}

An essential disadvantage of the derivation of the kinetic and interaction energies given in Sec. II is that the important microscopic information remains hidden in Eqs. (5), (17), and (18). This is why the consideration of Sec. II is below supplemented by the calculations of the pairwise-interaction contribution to $\varepsilon_{\text {int }}$ and $\varepsilon_{\text {kin }}$ via the in-medium pair wave functions (PWF's). Not to go into much detail but outline a physical picture, these calculations are limited to the leading order in $k_{F} a$.

For the sake of convenience, let us begin with the interaction energy. All the microscopic information concerning the $N$-particle system is contained in the $N$-particle density matrix. For the zero-temperature case this matrix is defined by

$$
\begin{aligned}
& \varrho_{N}\left(x_{1}^{\prime}, x_{2}^{\prime}, \ldots, x_{N}^{\prime} ; x_{1}, x_{2}, \ldots, x_{N}\right) \\
& \quad=\Psi *\left(x_{1}, x_{2}, \ldots, x_{N}\right) \Psi\left(x_{1}^{\prime}, x_{2}^{\prime}, \ldots, x_{N}^{\prime}\right),
\end{aligned}
$$

where $\Psi\left(x_{1}, x_{2}, \ldots, x_{N}\right)$ is the ground-state normalized wave function, $x=\{\mathbf{r}, \sigma\}$ stands for the space coordinates $\mathbf{r}$, and the spin $z$ projection $\sigma= \pm 1 / 2$. To investigate the interaction energy, we do not need to know the $N$-matrix in detail but can deal with the 2-matrix given by the integral

$$
\begin{aligned}
\varrho_{2}\left(x_{1}^{\prime}, x_{2}^{\prime} ; x_{1}, x_{2}\right)= & \int_{V} d x_{3} \cdots d x_{N} \Psi *\left(x_{1}, x_{2}, x_{3}, \ldots, x_{N}\right) \\
& \times \Psi\left(x_{1}^{\prime}, x_{2}^{\prime}, x_{3}, \ldots, x_{N}\right)
\end{aligned}
$$

where in general

$$
\int_{V} \cdots d x=\sum_{\sigma} \int_{V} \cdots d^{3} r
$$

Let us introduce the eigenfunctions of the 2-matrix $\xi_{\nu}\left(x_{1}, x_{2}\right)$ given by

$$
\int_{V} d x_{1} d x_{2} \varrho_{2}\left(x_{1}^{\prime}, x_{2}^{\prime} ; x_{1}, x_{2}\right) \xi_{\nu}\left(x_{1}, x_{2}\right)=w_{\nu} \xi_{\nu}\left(x_{1}^{\prime}, x_{2}^{\prime}\right)
$$

where $w_{\nu}$ stands for the $\nu$-state eigenvalue. These eigenfunctions are usually called in-medium PWF's [38]. The 2-matrix can be expressed in terms of its eigenfunctions and eigenvalues as follows:

$$
\varrho_{2}\left(x_{1}, x_{2} ; x_{1}^{\prime}, x_{2}^{\prime}\right)=\sum_{\nu} w_{\nu} \xi_{\nu}^{*}\left(x_{1}, x_{2}\right) \xi_{\nu}\left(x_{1}^{\prime}, x_{2}^{\prime}\right)
$$

where it is implied that

$$
\int_{V} d x_{1} d x_{2} \xi_{\nu}^{*}\left(x_{1}, x_{2}\right) \xi_{\nu^{\prime}}\left(x_{1}, x_{2}\right)=\delta_{\nu \nu^{\prime}}
$$

From Eq. (39) it follows that

$$
\int_{V} d x_{1} d x_{2} \varrho_{2}\left(x_{1}, x_{2} ; x_{1}, x_{2}\right)=1
$$

and, hence,

$$
\sum_{\nu} w_{\nu}=1
$$

which allows one to interprete the eigenvalue $w_{\nu}$ as the probability of observing a particle pair in the $\nu$ state.

Now let us recall that the total momentum of the system of interest, the total system spin, and its $z$ projection are conserved quantities [39]. As the total pair momentum $\hbar \hat{Q}$, the total pair spin $\hat{S}$, and its $z$ component $\hat{S}_{z}$ commute with the total system momentum, total system spin, and its $z$ projection, correspondingly, they commute with the system Hamiltonian given by Eq. (1), too. If so, then one can derive that $\hat{Q}, \hat{S}$, and $\hat{S}_{z}$ commute with the 2-matrix being permutable with the Hamiltonian (1). This is why we can choose the eigenfunctions of the 2-matrix in such a way that [38] $\nu$ $=\left\{\lambda, \mathbf{Q}, S, m_{S}\right\}$, where $m_{S}$ is an eigenvalue of $S_{z}$ and $\lambda$ stands for other quantum numbers. Hence, in the homogeneous situation one can arrive at (see Refs. $[38,40]$ )

$$
\xi_{\nu}\left(x_{1}, x_{2}\right)=\vartheta_{\nu}\left(\mathbf{r}, \sigma_{1}, \sigma_{2}\right) \exp (i \mathbf{Q} \cdot \mathbf{R}) / \sqrt{V},
$$

where $\mathbf{r}=\mathbf{r}_{1}-\mathbf{r}_{2}$ and $\mathbf{R}=\left(\mathbf{r}_{1}+\mathbf{r}_{2}\right) / 2$. As the in-medium bound pair states like the BCS pairs are beyond the scope of the present work, then we deal only with the scattering or "dissociated" pair states. Hence, $\nu=\left\{\mathbf{q}, \mathbf{Q}, S, m_{S}\right\}$, where q stands for the relative wave vector. This is why it is convenient to set by definition

$$
\vartheta_{\nu}\left(\mathbf{r}, \sigma_{1}, \sigma_{2}\right)=\varphi_{\nu}\left(\mathbf{r}, \sigma_{1}, \sigma_{2}\right) / \sqrt{V}
$$

as periodic boundary conditions are implied. Furthermore, the pairwise interaction does not depend on the spin variables, which means that $\varphi_{\nu}\left(\mathbf{r}, \sigma_{1}, \sigma_{2}\right)$ can be expressed as

$$
\varphi_{\nu}\left(\mathbf{r}, \sigma_{1}, \sigma_{2}\right)=\varphi_{\mathbf{q}, \mathbf{Q}, S}(\mathbf{r}) \chi_{S, m_{S}}\left(\sigma_{1}, \sigma_{2}\right)
$$

For the singlet states $(S=0)$ one gets

$$
\chi_{0,0}\left(\sigma_{1}, \sigma_{2}\right)=-\chi_{0,0}\left(\sigma_{2}, \sigma_{1}\right)
$$

and, due to the Fermi statistics,

$$
\varphi_{\mathbf{q}, \mathbf{Q}, 0}(\mathbf{r})=\varphi_{\mathbf{q}, \mathbf{Q}, 0}(-\mathbf{r})=\varphi_{-\mathbf{q}, \mathbf{Q}, 0}(\mathbf{r}),
$$

while for the triplet wave functions $(S=1)$,

$$
\chi_{1, m_{S}}\left(\sigma_{1}, \sigma_{2}\right)=\chi_{1, m_{S}}\left(\sigma_{2}, \sigma_{1}\right)
$$

and, hence, 


$$
\varphi_{\mathbf{q}, \mathbf{Q}, 1}(\mathbf{r})=-\varphi_{\mathbf{q}, \mathbf{Q}, 1}(-\mathbf{r})=-\varphi_{-\mathbf{q}, \mathbf{Q}, 1}(\mathbf{r}) .
$$

For $r \rightarrow \infty$ we have

$$
\varphi_{\mathbf{q}, \mathbf{Q}, 0}(\mathbf{r}) \rightarrow \sqrt{2} \cos (\mathbf{q} \cdot \mathbf{r}),
$$

whereas

$$
\varphi_{\mathbf{q}, \mathbf{Q}, 1}(\mathbf{r}) \rightarrow \sqrt{2} \sin (\mathbf{q} \cdot \mathbf{r}) .
$$

Working in the thermodynamic limit $N \rightarrow \infty, V \rightarrow \infty$, $N / V=n=$ const, it is more convenient to leave the 2-matrix in favor of the so-called pair correlation function

$$
F_{2}\left(x_{1}, x_{2} ; x_{1}^{\prime}, x_{2}^{\prime}\right)=\left\langle\hat{\psi}^{\dagger}\left(x_{1}\right) \hat{\psi}^{\dagger}\left(x_{2}\right) \hat{\psi}\left(x_{2}^{\prime}\right) \hat{\psi}\left(x_{1}^{\prime}\right)\right\rangle,
$$

where $\langle\hat{A}\rangle$ stands for the statistical average of the operator $\hat{A}$, and $\hat{\psi}^{\dagger}(x)$ and $\hat{\psi}(x)$ denote the field Fermi operators. The pair correlation function differs from the 2-matrix by the normalization factor (see Ref. [38])

$$
F_{2}\left(x_{1}, x_{2} ; x_{1}^{\prime}, x_{2}^{\prime}\right)=N(N-1) \varrho_{2}\left(x_{1}^{\prime}, x_{2}^{\prime} ; x_{1}, x_{2}\right),
$$

so that $F_{2}$ remains finite while $\varrho_{2}$ approaches zero in the thermodynamic limit. Indeed, when $V \rightarrow \infty, N \rightarrow \infty, N / V=n$ $\rightarrow$ const, Eqs. (41) and (54), taken in conjunction with Eqs. (44) and (45), yield

$$
\begin{aligned}
F_{2}\left(x_{1}, x_{2} ; x_{1}^{\prime}, x_{2}^{\prime}\right) & \\
= & \sum_{S, m_{S}} \int \frac{d^{3} q d^{3} Q}{(2 \pi)^{6}} \rho_{S, m_{S}}(\mathbf{q}, \mathbf{Q}) \varphi_{\mathbf{q}, \mathbf{Q}, S}^{*}(\mathbf{r}) \varphi_{\mathbf{q}, \mathbf{Q}, S}\left(\mathbf{r}^{\prime}\right) \\
& \quad \times \chi_{S, m_{S}}^{*}\left(\sigma_{1}, \sigma_{2}\right) \chi_{S, m_{S}}\left(\sigma_{1}^{\prime}, \sigma_{2}^{\prime}\right) \exp \left\{i \mathbf{Q}\left(\mathbf{R}^{\prime}-\mathbf{R}\right)\right\},
\end{aligned}
$$

where the momentum-distribution function

$$
\rho_{S, m_{S}}(\mathbf{q}, \mathbf{Q})=\lim _{V, N \rightarrow \infty}\left\{N(N-1) w_{\mathbf{q}, \mathbf{Q}, S, m_{S}}\right\}
$$

is finite because $w_{\mathbf{q}, \mathbf{Q}, S, m_{S}} \sim 1 / V^{2}$ [this follows from Eq. (43) when $V \rightarrow \infty]$. For $\rho_{S, m_{S}}(\mathbf{q}, \mathbf{Q})$ one gets the relation

$$
\sum_{S, m_{S}} \int \frac{d^{3} q d^{3} Q}{(2 \pi)^{6}} \rho_{S, m_{S}}(\mathbf{q}, \mathbf{Q})=n^{2},
$$

resulting from Eqs. (43) and (56).

All the necessary formulas are now discussed and displayed, and we can turn to the calculations of the interaction energy. Using the expression (see the textbook [28])

$$
E_{\text {int }}=\frac{1}{2} \int d x_{1} d x_{2} V\left(\left|\mathbf{r}_{1}-\mathbf{r}_{2}\right|\right) F_{2}\left(x_{1}, x_{2} ; x_{1}, x_{2}\right)
$$

and keeping in mind Eq. (55), one gets the following important relation:

$$
\varepsilon_{\mathrm{int}}=\frac{1}{2 n} \int d^{3} r V(r) \sum_{S, m_{S}} \int \frac{d^{3} q d^{3} Q}{(2 \pi)^{6}} \rho_{S, m_{S}}(\mathbf{q}, \mathbf{Q})\left|\varphi_{\mathbf{q}, \mathbf{Q}, S}(\mathbf{r})\right|^{2},
$$

provided the equality

$$
\sum_{\sigma_{1}, \sigma_{2}} \chi_{S, m_{S}}^{*}\left(\sigma_{1}, \sigma_{2}\right) \chi_{S, m_{S}}\left(\sigma_{1}, \sigma_{2}\right)=1
$$

is taken into account. Equation (59) directly connects the interaction energy per fermion with PWF's and, thus, with the scattering waves defined by

$$
\psi_{\mathbf{q}, \mathbf{Q}, 0}(\mathbf{r})=\varphi_{\mathbf{q}, \mathbf{Q}, 0}(\mathbf{r})-\sqrt{2} \cos (\mathbf{q} \cdot \mathbf{r})
$$

and

$$
\psi_{\mathbf{q}, \mathbf{Q}, 1}(\mathbf{r})=\varphi_{\mathbf{q}, \mathbf{Q}, 1}(\mathbf{r})-\sqrt{2} \sin (\mathbf{q} \cdot \mathbf{r}) .
$$

The scattering waves are immediately related to the pairwisepotential contribution to the spatial particle correlations. Setting $\psi_{\mathbf{q}, \mathbf{Q}, 0}(\mathbf{r})=\psi_{\mathbf{q}, \mathbf{Q}, 1}(\mathbf{r})=0$ or, in other words, ignoring that contribution and taking notice only of the correlations due to the statistics, one arrives at the Hartree-Fock scheme.

So far we did not invoke any approximation when operating with the 2-matrix and pair correlation function [41]. However, taken in the regime of a dilute Fermi gas, Eq. (59) can significantly be simplified. Indeed, the lower the densities, the lower the momenta are typical of the system. This means that the pair momentum distribution $\rho_{S, m_{S}}(\mathbf{q}, \mathbf{Q})$ is getting more localized in a small vicinity of the point $q=Q$ $=0$ when $n \rightarrow 0$. Consequently, the low-momentum approximation can be applied according to which for $n \rightarrow 0$ we get

$$
\int \frac{d^{3} q d^{3} Q}{(2 \pi)^{6}} \rho_{S, m_{S}}(\mathbf{q}, \mathbf{Q})\left|\varphi_{\mathbf{q}, \mathbf{Q}, S}(\mathbf{r})\right|^{2} \simeq\left|\varphi_{S}(\mathbf{r})\right|^{2} \eta_{S, m_{S}},
$$

where

$$
\eta_{S, m_{S}}=\int \frac{d^{3} q d^{3} Q}{(2 \pi)^{6}} \rho_{S, m_{S}}(\mathbf{q}, \mathbf{Q})
$$

and

$$
\varphi_{S}(\mathbf{r})=\lim _{\mathbf{q}, \mathbf{Q} \rightarrow 0} \varphi_{\mathbf{q}, \mathbf{Q}, S}(\mathbf{r}) .
$$

From Eqs. (50) and (64) it follows that $\varphi_{S=1}(\mathbf{r})=0$. This result, taken in conjunction with the low-momentum approximation of Eq. (62), makes it possible to conclude that Eq. (59) reduces for $n \rightarrow 0$ to

$$
\varepsilon_{\mathrm{int}} \simeq \frac{\eta_{0,0}}{2 n} \int d^{3} r V(r)\left|\varphi_{0}(\mathbf{r})\right|^{2}
$$

The triplet states do not make any contribution to the interaction energy in the approximation (62), and this completely meets the usual expectations.

Now, to employ Eq. (65), one should have an idea concerning $\varphi_{0}(\mathbf{r})$ and $\eta_{0,0}$. As to the limiting wave function $\varphi_{0}(\mathbf{r})$, it can be determined by means of the following simple and custom arguments. In the dilution limit the pair wave function $\varphi_{\mathbf{q}, \mathbf{Q}, S}(\mathbf{r})$ approaches the solution of the ordinary Schrödinger equation

$$
-\left(\hbar^{2} / m\right) \nabla^{2} \varphi_{\mathbf{q}, \mathbf{Q}, S}(\mathbf{r})+V(r) \varphi_{\mathbf{q}, \mathbf{Q}, S}(\mathbf{r})=\left(\hbar^{2} q^{2} / m\right) \varphi_{\mathbf{q}, \mathbf{Q}, S}(\mathbf{r}),
$$

with the boundary conditions given by Eqs. (48) and (50). Hence, in the limit $n \rightarrow 0$ the quantity $\varphi_{0}(r)$ obeys Eq. (11) 
and $\varphi_{0}(\mathbf{r}) \rightarrow \sqrt{2}$ when $r \rightarrow \infty$. Therefore, for $n \rightarrow 0$ one gets

$$
\varphi_{0}(\mathbf{r}) \simeq \sqrt{2} \varphi(r) .
$$

To complete the calculation of the interaction energy, it only remains to find $\eta_{0,0}$. One can expect that when numbers of fermions with positive and negative spin $z$ projections are the same, the magnitude of $\rho_{S, m_{S}}(\mathbf{q}, \mathbf{Q})$ appears to be independent of the spin variables. In this case Eqs. (57) and (63) give

$$
\eta_{S, m_{S}}=n^{2} / 4 \text {. }
$$

Note that Eq. (68) can be found in a more rigorous way with the relation

$$
\frac{1}{V^{2}} \int d^{3} r_{1} d^{3} r_{2} F_{2}\left(x_{1}, x_{2} ; x_{1}, x_{2}\right)=n_{\sigma_{1}} n_{\sigma_{2}}
$$

resulting from the definition of the pair correlation function (53). Here $n_{\sigma}=\left\langle\hat{\psi}^{\dagger}\left(x_{1}\right) \hat{\psi}\left(x_{1}\right)\right\rangle$. To derive Eq. (68) from Eq. (69), one should employ the latter in conjunction with the explicit form of $\chi_{S, m_{S}}\left(\sigma_{1}, \sigma_{2}\right)$ (see, for example, Ref. [40]) and Eq. (55) and, then, take account of $n_{\sigma}=\left\langle\hat{\psi}^{\dagger}\left(x_{1}\right) \hat{\psi}\left(x_{1}\right)\right\rangle$ $=n / 2$. Let us stress that Eq. (68) is not general. For example, when all the considered fermions have the spin $z$ projection equal to $+1 / 2$, one gets $\eta_{1,1}=n^{2}$ and $\eta_{0,0}=\eta_{1,-1}=\eta_{1,0}=0$. As is seen, in this case the interaction energy resulting from Eq. (65) exactly amounts to zero: one should go beyond the approximation defined by Eq. (62) to get an idea about $\varepsilon_{\text {int }}$ of such a weakly interacting system. Here it is worth noting that this weak interaction is a serious obstacle that prevents experimentalists from observing the BCS-like pairing of fermions due to an extremely low temperature of the BCS transition. To strengthen the interaction effects, it was, in particular, suggested [15] to complicate the experimental scheme in such a way that fermions with various spin $z$ projections would be trapped. In this case the low-momentum approximation yields a finite result for $\varepsilon_{\text {int }}$. It is instructive to go in a more detail about this situation because now it is under experimental investigation [14]. Here another choice of the eigenfunctions of the 2-matrix is more convenient rather than that of Eq. (46). The details can be found in the Appendix.

At last, inserting Eqs. (67) and (68) into Eq. (65) and making use of Eqs. (14) and (16), one can derive $\varepsilon_{\text {int }}$ $=\pi \hbar^{2} n(a-b) / m$, which is the leading term of Eq. (18). It is worth noting that to calculate the next orders of Eq. (18) by means of PWF's, one should employ a more accurate procedure similar to that developed for a dilute Bose gas in Refs. $[19,20]$. The important point of such a procedure is taking account of the in-medium corrections to PWF. Though a complex investigation of this problem is beyond the scope of the present work, several remarks about the medium effects on PWF's are made in the next section.

Now it is interesting to check if Eq. (65) yields Eq. (25) when replacing $V(r)$ by the pseudopotential $V^{(\mathrm{ps})}(r)$. The simplest way of operating with the pseudopotential is to adopt $V^{(\mathrm{ps})}(r)=\left(4 \pi \hbar^{2} a / m\right) \delta(\mathbf{r})$ in conjunction with the Hartree-Fock approximation. It allows for calculating the contribution of the pairwise interaction to the total energy of a ground-state quantum gas in the leading order of the expansion in the dilution parameter. For example, this way was used in the classical paper by Pitaevskii when deriving the Gross-Pitaevskii equation for the order parameter of the Bose-Einstein condensation in a dilute Bose gas [4]. A more elaborated variant, which goes beyond the Hartree-Fock framework, requires a more sophisticated choice of pseudopotential given by $V^{(\mathrm{ps})}(r)=\left(4 \pi \hbar^{2} a / m\right) \delta(\mathbf{r})(\partial / \partial r) r$ [25]. This variant makes it possible to calculate the contribution of the pairwise interaction to the total energy in the both leading and next-to-leading orders in the dilution parameter. To provide an illustration, we can limit ourselves to the simplest choice of pseudopotential. Replacing $V(r)$ by $V^{(\mathrm{ps})}(r)$ $=\left(4 \pi \hbar^{2} a / m\right) \delta(\mathbf{r})$ in Eq. (65) and setting $\psi(r)=0[\varphi(r)=1]$, one can find $\varepsilon_{\text {int }}^{(\mathrm{ps})}=\pi \hbar^{2} a n / m$, which is exactly Eq. (25) taken to the leading order in $k_{F} a$. This supports the conclusion of Sec. III about the serious limitations of the pseudopotential procedure due to the wrong results for the kinetic and interaction energies.

As was mentioned in Sec. III, the pseudopotential approach does not yield a correct picture of the short-range boson correlations in addition to the failure with the kinetic and interaction energies in the case of a dilute Bose gas [19]. The same fault can be expected in the Fermi case, as well. In view of this fact, it is important to remark that the procedure based on PWF's yields a correct picture of the short-range particle correlations. Indeed, from Eq. (55) it follows that the pair distribution function $g(r)$, defined by $g(r)$ $=\left(1 / n^{2}\right) \sum_{\sigma_{1}, \sigma_{2}} F_{2}\left(x_{1}, x_{2} ; x_{1}, x_{2}\right)$, is expressed as

$$
g(r)=\frac{1}{n^{2}} \int \frac{d^{3} q d^{3} Q}{(2 \pi)^{6}} \rho_{S, m_{S}}(\mathbf{q}, \mathbf{Q})\left|\varphi_{\mathbf{q}, \mathbf{Q}, S}(\mathbf{r})\right|^{2} .
$$

When using the low-momentum approximation in conjunction with Eqs. (67), (68), and (70), Eq. (70) is reduced to

$$
g(r) \simeq(1 / 2) \varphi^{2}(r),
$$

which is correct at $n \rightarrow 0$ and for $k_{F} r \ll 1$. This short-range result differs from the corresponding result for a dilute Bose gas $[19,20]$ by a factor of $1 / 2$ appearing due to the Fermi statistics. Note that this factor manifests itself in the total energy, as well. Indeed, the leading term in the dilution expansion for the total energy of a ground-state uniform 3D Bose gas is twice more than the first $a$-dependent term in the corresponding expansion for the Fermi gas [28]. Contrary to the results of the calculations based on the pseudopotential (see Ref. [19]), there are no negative values of $g(r)$ at small particle separations when using the 2-matrix.

Several remarks need to be added concerning the momentum distribution of the "dissociated" pairs $\rho_{S, m_{S}}(\mathbf{q}, \mathbf{Q})$. The calculations resulting in Eq. (65) do not involve detailed knowledge of this distribution. However, its form can be completely refined. As was shown in Ref. [40], $\rho_{S, m_{S}}(\mathbf{q}, \mathbf{Q})$ can be determined via the correlation-weakening principle. According to this principle the pair correlation function obeys the following relation:

$$
F_{2}\left(x_{1}, x_{2} ; x_{1}^{\prime}, x_{2}^{\prime}\right) \rightarrow F_{1}\left(x_{1} ; x_{1}^{\prime}\right) F_{1}\left(x_{2} ; x_{2}^{\prime}\right),
$$

when 


$$
\left|\mathbf{r}_{1}-\mathbf{r}_{2}\right| \rightarrow \infty, \quad\left|\mathbf{r}_{1}-\mathbf{r}_{1}^{\prime}\right|=\text { const, } \quad\left|\mathbf{r}_{2}-\mathbf{r}_{2}^{\prime}\right|=\text { const } .
$$

In Eq. (72) we set $F_{1}\left(x_{1} ; x_{1}^{\prime}\right)=\left\langle\hat{\psi}^{\dagger}\left(x_{1}\right) \hat{\psi}\left(x_{1}^{\prime}\right)\right\rangle$. So the pair momentum distribution $\rho_{S, m_{S}}(\mathbf{q}, \mathbf{Q})$, which appears in the expansion of $F_{2}$ in the set of its eigenfunctions, can be expressed in terms of the single-particle momentum distribution $n_{\sigma}(k)$ $=\left\langle a_{\sigma}^{\dagger}(\mathbf{k}) a_{\sigma}(\mathbf{k})\right\rangle$, which comes into the plane-wave expansion for $F_{1}$. In the case of interest, when the both distribution functions turn out to be independent of spin variables, this leads to

$$
\rho_{S, m_{S}}(\mathbf{q}, \mathbf{Q})=n(|\mathbf{Q} / 2+\mathbf{q}|) n(|\mathbf{Q} / 2-\mathbf{q}|),
$$

where, by definition, $n(k)=n_{\sigma}(k)$. Concluding let us set, for the sake of demonstration, $n(k)=1$ for $k \leqslant k_{F}, n(k)=0$ for $k$ $>k_{F}$ and return to Eq. (62). Inserting Eq. (73) in the righthand side of Eq. (62) and utilizing this single-particle momentum distribution of an ideal Fermi gas, we arrive at the left-hand side of Eq. (62) due to $k_{F} \rightarrow 0$ when $n \rightarrow 0$.

Thus, in Sec. IV the interaction energy of the Fermi gas was calculated to the leading order in $k_{F} a$ "from scratch." These calculations yield a result being in agreement with Eq. (18) and provide detailed information about the contribution of the pairwise interaction to $\varepsilon_{\text {int }}$. Now it is of interest to display the "mediator" between the pairwise potential and kinetic energy. A discussion of this subject is given in Sec. V.

\section{KINETIC ENERGY VIA THE PAIR WAVE FUNCTIONS}

Some hints about the way of treating the problem of the influence of the pairwise interaction on the kinetic energy can be selected in the Bogoliubov model of a weakly interacting Bose gas and in the BCS model. As was shown in Refs. [19,20], there exists an important relation connecting the single-boson momentum distribution $n_{B}(k)$ with the scattering parts of PWF's in the Bogoliubov model. For the case of the ground state this relation is written as

$$
n_{B}(k)\left[1+n_{B}(k)\right]=n_{0}^{2}\left|\psi_{B}(k)\right|^{2},
$$

where $\psi_{B}(k)$ is the Fourier transform for the scattering part of the bosonic PWF's corresponding to $\mathbf{q}=\mathbf{Q}=0$, and $n_{0}$ stands for the density of the condensed bosons. Note that the PWF's with $\mathbf{q}, \mathbf{Q} \neq 0$ are the plane waves in the Bogoliubov model $[19,20]$. When the pairwise boson interaction is being "switched off," $\psi_{B}(k)$ approaches zero. At $\psi_{B}(k)=0$, Eq. (74) yields the only physical solution $n_{B}(k)=0$, which corresponds to the ideal Bose gas with the zero condensate depletion and kinetic energy. On the contrary, "switching on" the pairwise interaction results in $\psi_{B}(k) \neq 0$. Due to Eq. (74), this leads to $n_{B}(k) \neq 0$ and to the kinetic energy dependent on $V(r)$.

A similar relation appears in the BCS model. At the zero temperature it can be represented [40] in the form

$$
n_{\mathrm{BCS}}(k)\left[1-n_{\mathrm{BCS}}(k)\right]=n_{C}^{2}\left|\vartheta_{\mathrm{BCS}}(k)\right|^{2},
$$

where $n_{\mathrm{BCS}}(k)$ stands for the single-fermion distribution, $\vartheta_{\mathrm{BCS}}(k)$ is the Fourier transform of the Cooper-pair wave function, and $n_{C}$ is the density of the Cooper pairs. We remark that the "dissociated" pair states are the plane waves in the framework of the BCS approach [40]. "Switching off" the pairwise interaction leads to the disappearance of the in-medium bound states: $\vartheta_{\mathrm{BCS}}(k)=0$. In this case there exist two branches of the solution of Eq. (75): $n_{\mathrm{BCS}}=1$ and $n_{\mathrm{BCS}}$ $=0$. The system acquires minimal energy if selecting the first branch below the Fermi momentum and taking the second one above, which yields the momentum distribution of the ideal Fermi gas. When "switching on" the mutual attraction of fermions, this momentum distribution acquires corrections due to the right-hand side of Eq. (75).

Now, keeping in mind these examples, we can assume that the relation connecting PWF (more precisely, the scattering parts and bound pair waves) with the single-particle momentum distribution is a general feature of any quantum many-body system. If so, exactly this relation should be responsible for the influence of the pairwise interaction on the kinetic energy of the Fermi gas. For the situation in question it can be constructed as

$$
n(k)[1-n(k)]=\mathcal{L}(k),
$$

where $\mathcal{L}(k)$ stands for a functional of $\psi_{\mathbf{q}, \mathbf{Q}, S}(\mathbf{r})$. To go in a more detail about $\mathcal{L}(k)$, let us calculate the kinetic energy, operating with $n(k)$ of Eq. (76). From Eq. (76) it follows that

$$
n(k)=\left\{\begin{array}{lll}
{[1+\sqrt{1-4 \mathcal{L}(k)}] / 2} & \text { if } & k \leqslant \mathcal{K}_{F}, \\
{[1-\sqrt{1-4 \mathcal{L}(k)}] / 2} & \text { if } & k>\mathcal{K}_{F},
\end{array}\right.
$$

where $\hbar \mathcal{K}_{F}$ stands for the Fermi momentum in the presence of the pairwise interaction. The momentum distribution $n(k)$ approaches the Fermi distribution in the dilution limit: $\mathcal{L}(k) \rightarrow 0, \mathcal{K}_{F} \rightarrow k_{F}$ when $n \rightarrow 0$. Then, for $n \rightarrow 0$, Eq. (77) is reduced to

$$
n(k)=[1-\mathcal{L}(k)] \Theta\left(k_{F}-k\right)+\mathcal{L}(k) \Theta\left(k-k_{F}\right) .
$$

Taken together with the familiar formula

$$
\varepsilon_{\mathrm{kin}}=\sum_{\sigma} \int \frac{d^{3} k}{(2 \pi)^{3}} T_{k} n_{\sigma}(k),
$$

Eq. (78) results in

$$
\varepsilon_{\text {kin }}=\frac{3 \hbar^{2} k_{F}^{2}}{10 m}+\frac{2}{n} \int \frac{d^{3} k}{(2 \pi)^{3}} T_{k} \ell(k)+\cdots .
$$

Comparing Eq. (17) with Eq. (80), we arrive at

$$
\mathcal{L}(k) \rightarrow\left(n^{2} / 4\right) \psi^{2}(k) \quad(n \rightarrow 0) .
$$

This suggests that $\mathcal{L}(k)$ is indeed a functional of $\psi_{\mathbf{p}, \mathbf{q}, S}(\mathbf{r})$.

It is worth noting that substitution of the right-hand side of Eq. (81) for $\mathcal{L}(k)$ in Eq. (78) yields a good approximation for $n(k)$ only when $k \gtrsim k_{F}$. From Eq. (11) it follows that $\psi(k) \propto-1 / k^{2}$ for $k \rightarrow 0$, which prevents us from using this substitution in the long-wavelength regime. Hence, to consider $n(k)$ for $k \rightarrow 0$, one should go beyond Eq. (81). In particular, keeping in mind the replacement of $\varphi_{0}(r)$ by $\sqrt{2} \varphi(r)$ in Eq. (65), we can introduce the approximation 


$$
\mathcal{L}(k) \approx\left(n^{2} / 8\right)\left|\psi_{0}(k)\right|^{2},
$$

where $\psi_{0}(k)$ is the Fourier transform of $\psi_{0}(r)=\varphi_{0}(r)-\sqrt{2}$. To have an idea about Eq. (76) taken together with Eq. (82), it is natural to start from the expectation that the Fermi sphere is completely occupied. In this case Eqs. (76) and (82) lead to $\psi_{0}(k)=0$ at $k \leqslant \mathcal{K}_{F} \simeq k_{F}$. In the region of high fermion momenta (reflecting the physics at small particle separations), $\psi_{0}(k)$ is not significantly influenced by the surrounding medium and, therefore, is nearly governed by the ordinary twobody Schrödinger equation taken in the center-of-mass frame and at the zero energy. Combination of these two regimes results in

$$
2 T_{k} \psi_{0}(k)+\Theta\left(k-k_{F}\right) \int d^{3} r \varphi_{0}(r) V(r) \exp (-i \mathbf{k} \cdot \mathbf{r})=0 .
$$

Note that a jump in the Fourier transform of $\psi_{0}(r)$ at $k=k_{F}$ does not imply a jump of $\psi_{0}(r)$ itself. Passing to the spacecoordinate representation, one is able to rewrite Eq. (83) in the form

$$
-\frac{\hbar^{2}}{m} \nabla^{2} \varphi_{0}(r)+\varphi_{0}(r) V(r)=\int d^{3} r^{\prime} \varphi_{0}\left(r^{\prime}\right) V\left(r^{\prime}\right) G\left(\mathbf{r}-\mathbf{r}^{\prime}\right),
$$

where $G(\mathbf{r})=\int_{k<k_{F}} d^{3} k /(2 \pi)^{3} \exp [i \mathbf{k} \cdot \mathbf{r}]$. Equation (84) is nothing more but the simplest version of the BetheGoldstone equation [26] for the pair wave function at $\mathbf{Q}=\mathbf{q}$ $=0$. Hence, the relation between the fermion momentum distribution and scattering parts of PWF's is directly connected with the familiar "veto" upon the appearance of the intermediate scattering states inside the Fermi sphere (see the p. 320 in the textbook in [28]). We can now turn to a more accurate way of treating $\varphi_{0}(r)$. The total system energy can be represented as a functional of $\varphi_{0}(r)$ and $n(k)$ with the help of Eqs. (65) and (79). Making a variation of this functional with respect to $\psi_{0}^{*}(r)$ and $n(k)$, one gets

$$
\begin{aligned}
\frac{\delta E}{V}= & \int \frac{d^{3} k}{(2 \pi)^{3}}\left[2 T_{k} \delta n(k)+\frac{n^{2}}{8} \delta \psi_{0}^{*}(k)\right. \\
& \left.\times \int d^{3} r \varphi_{0}(r) V(r) \exp (-i \mathbf{k} \cdot \mathbf{r})\right] .
\end{aligned}
$$

The infinitesimal changes $\delta n(k)$ and $\delta \psi_{0}^{*}(k)$ are not independent but related to one another by

$$
[1-2 n(k)] \delta n(k)=\left(n^{2} / 8\right) \psi_{0}(k) \delta \psi_{0}^{*}(k),
$$

which follows from Eq. (76). In addition, the total number of fermions, $N=2 \int d^{3} k /(2 \pi)^{3} n(k)$, should not be changed so that the equation of interest is written in the form

$$
\delta(E-\mu N)=0,
$$

where $\mu$ stands for the chemical potential. Equations (85)-(87) make it possible to arrive at

$$
\psi_{0}(k)=\frac{1-2 n(k)}{2\left(\mu-T_{k}\right)} \int d^{3} r \varphi_{0}(r) V(r) \exp (-i \mathbf{k} \cdot \mathbf{r}) .
$$

This equation is more complicated but similar to Eq. (83). Indeed, for $k \rightarrow \infty$, Eq. (88) approaches the two-body Schrödinger equation taken in the center-of-mass frame at zero energy, whereas the contribution of the mediumdependent terms is significant only for $k \leqq \mathcal{K}_{F}$. However, the important difference is that $\psi_{0}(k)$ is now suppressed but does not vanish inside the Fermi sphere. This is due the fact that the Fermi sphere is not completely occupied in the presence of the pairwise interaction. It is worth noting that the equation derived by Galitskii [27] for the scattering part of the "effective wave function of two particles in a medium" [see Eq. (11.39) in Ref. [28]] is exactly reduced to Eq. (88) when $\mathbf{Q}, \mathbf{q} \rightarrow 0$. To the best knowledge of the present author, Galitskii did not associate the "effective wave functions" with the eigenfunctions of the 2-matrix but introduced this name due to the similarity with the two-body Schrödinger equation. However, the derived result suggests that the Galitskii "effective wave functions" are actually eigenfunctions of the reduced density matrix of the second order.

Thus, it was shown in Sec. V that the "mediator" between the pairwise interaction and kinetic energy of a ground-state uniform repulsive 3D Fermi gas is the relation connecting the single-particle momentum distribution with the scattering parts of PWF's. A similar relation takes place for the uniform ground-state 2D and 3D Bose gases, which results in the appearance of a condensate depletion and nonzero kinetic energy in the presence of the repulsive pairwise interaction. The mutual attraction of particles has also an effect on the momentum distribution and kinetic energy of the quantum gases. However, in this case the scattering parts of PWF's are accompanied by the in-medium bound states. In general, one can expect that influence of the pairwise interaction on the kinetic energy of any quantum many-body system is governed by the relation connecting the single-particle momentum distribution with PWF's.

\section{CONCLUSION}

In conclusion, the kinetic and interaction energies of a uniform ground-state 3D Fermi gas with a repulsive shortrange pairwise interaction have been calculated up to the second order in the gas parameter $k_{F} a$. These quantities were found to depend on the pairwise potential through the two characteristic lengths. One of them is the $s$-wave scattering length $a$ which also appears in the total energy. Another, $b$ defined by Eq. (16), makes contributions only to the kinetic and interaction energies taken separately: the terms depending on $b$ are mutually canceled in the total energy in the first three orders of the expansion in $k_{F} a$. The derived results suggest that when the pairwise potential combines a hardsphere core with an attractive "tail" (in particular, this is relevant for the alkali-metal atoms), the Fermi gas turns out to be an interacting system even at $a=0$ though its total energy is equal to that of an ideal Fermi gas. In this case $b$ $\neq 0$, which results in a nonzero contribution of the pairwise interaction to both the kinetic and interaction energies. An- 
other interesting situation concerns the resonant regime $a$ $\rightarrow \infty$. The contribution of the pairwise potential to the kinetic energy near a point of the Fesbasch resonance is much larger than the corresponding contribution to the total energy. This is due to the fact that the interaction energy is negative in this case in spite of a positive scattering length.

Thus, contrary to a classical imperfect gas, the pairwise interaction has a nontrivial effect on the kinetic energy of quantum gases. Experiments with trapped alkali-metal vapors make it possible to check this effect, for example, by means of the standard technique based on time-of-flight expansion images $[1,2]$ introduced to probe the velocity distribution in a trapped atom cloud.

\section{ACKNOWLEDGMENTS}

It is a pleasure to acknowledge helpful discussions with colleagues at TFVS (Antwerpen Universiteit): J. T. Devreese, F. Brosens, and J. Tampere. The author thanks V. P. Mineev, E. Kats, M. E. Zhitomirskii, and G. Jackeli for kind hospitality and stimulating discussions during his visit to ILL and SPSMS (Grenoble). Fruitful interactions with A. Yu. Cherny and A. V. Vagov are acknowledged. The author thanks P. Naidon and F. Masnou-Seeuws for interest and hospitality during his visit to Laboratoire Aimé Cotton, SNRS (Orsay). This work was partly supported by RFBR Grant No. 00-02-17181.

\section{APPENDIX}

Let us consider a uniform cold 3D Fermi gas made of particles with the spin $\ell / 2(\ell>1$ is an odd number), the fermions with only two spin $z$ projections being present, say, $\ell / 2$ and $\ell / 2-\ell^{\prime}$, where $\ell^{\prime}$ is an integer and $\ell^{\prime} \leqslant \ell$. This situation may be of interest due to the recent experiments [14] with the atoms of the fermionic isotope ${ }^{40} \mathrm{~K}$ in an optical trap. The total atomic spin is $f=9 / 2$ for ${ }^{40} \mathrm{~K}$, and the incoherent mixture of $|9 / 2,-7 / 2\rangle$ and $|9 / 2,-9 / 2\rangle$ spin states was investigated in the experiments. In the case under consideration it is more convenient to choose a set of eigenfunctions of the 2-matrix in a way different from Sec. IV. We now choose $\nu=\left\{\mathbf{q}, \mathbf{Q}, S_{z}, \delta_{S_{z}}\right\}$, where $S_{z}$ stands for the eigenvalues of $\hat{S}_{z}$, the $z$ projection of the total pair spin, while $\delta_{S_{z}}$ enumerates degenerate states [for more detail, see Eq. (A2) below]. To simplify the calculations, we do not operate with the total pair spin here. Note that the same choice of the eigenfunctions could be used in Sec. IV. Equation (46) is now of the form

$$
\varphi_{\nu}\left(\mathbf{r}, \sigma_{1}, \sigma_{2}\right)=\varphi_{\mathbf{q}, \mathbf{Q}}^{(\delta)}(\mathbf{r}) \chi_{S_{z}}^{(\delta)}\left(\sigma_{1}, \sigma_{2}\right),
$$

where the spin variable $\sigma_{i}(i=1,2)$ takes the values $\ell / 2$ and $\ell / 2-\ell^{\prime}$. In Eq. (A1) the condensed notation $\delta=\delta_{S_{7}}$ is introduced, and $\delta$ is taken as a superscript, to stress the fact that it is an auxiliary quantity rather than important physical characteristics. The spin states are specified by

$$
\left|\chi_{S_{z}=\ell}^{(\delta=1)}\right\rangle=\left|\frac{\ell}{2}\right\rangle\left|\frac{\ell}{2}\right\rangle,
$$

$$
\begin{gathered}
\left|\chi_{S_{z}=\ell-\ell^{\prime}}^{(\delta=1)}\right\rangle=\frac{1}{\sqrt{2}}\left(\left|\frac{\ell}{2}\right\rangle\left|\frac{\ell}{2}-\ell^{\prime}\right\rangle+\left|\frac{\ell}{2}-\ell^{\prime}\right\rangle\left|\frac{\ell}{2}\right\rangle\right), \\
\left|\chi_{S_{z}=\ell-\ell^{\prime}}^{(\delta=2)}\right\rangle=\frac{1}{\sqrt{2}}\left(\left|\frac{\ell}{2}\right\rangle\left|\frac{\ell}{2}-\ell^{\prime}\right\rangle-\left|\frac{\ell}{2}-\ell^{\prime}\right\rangle\left|\frac{\ell}{2}\right\rangle\right), \\
\left|\chi_{S_{z}=\ell-2 \ell^{\prime}}^{(\delta=1)}\right\rangle=\left|\frac{\ell}{2}-\ell^{\prime}\right\rangle\left|\frac{\ell}{2}-\ell^{\prime}\right\rangle .
\end{gathered}
$$

There is no degeneracy for $S_{z}=\ell$ and $S_{z}=\ell-2 \ell^{\prime}$, and for these states $\delta_{S_{z}}=1$. While for $S_{z}=\ell-\ell^{\prime}$ two possible eigenstates of $\hat{S}_{z}$ are present, which is expressed in $\delta_{\ell-\ell^{\prime}}=1,2$. From Eq. (A2) it follows that

$$
\chi_{S_{z}}^{(1)}\left(\sigma_{1}, \sigma_{2}\right)=\chi_{S_{z}}^{(1)}\left(\sigma_{2}, \sigma_{1}\right),
$$

and, due to the Fermi statistics, we find

$$
\varphi_{\mathbf{q}, \mathbf{Q}}^{(1)}(\mathbf{r})=-\varphi_{\mathbf{q}, \mathbf{Q}}^{(1)}(-\mathbf{r})=-\varphi_{-\mathbf{q}, \mathbf{Q}}^{(1)}(\mathbf{r}),
$$

the wave function $\varphi_{\mathbf{q}, \mathbf{Q}}^{(1)}(\mathbf{r})$ approaching $\sqrt{2} \sin (\mathbf{q} \cdot \mathbf{r})$ when $r$ $\rightarrow \infty$, whereas for $\delta=2$ one gets

$$
\chi_{S_{z}}^{(2)}\left(\sigma_{1}, \sigma_{2}\right)=-\chi_{S_{z}}^{(2)}\left(\sigma_{2}, \sigma_{1}\right)
$$

and, hence,

$$
\varphi_{\mathbf{q}, \mathbf{Q}}^{(2)}(\mathbf{r})=\varphi_{\mathbf{q}, \mathbf{Q}}^{(2)}(-\mathbf{r})=\varphi_{-\mathbf{q}, \mathbf{Q}}^{(2)}(\mathbf{r}),
$$

where $\varphi_{\mathbf{q}, \mathbf{Q}}^{(2)}(\mathbf{r})$ tends to $\sqrt{2} \cos (\mathbf{q} \cdot \mathbf{r})$ for $r \rightarrow \infty$. Taken in conjunction with Eqs. (41), (44), and (45), Eq. (A1) yields

$$
\begin{aligned}
F_{2}\left(x_{1}, x_{2} ; x_{1}, x_{2}\right)= & \sum_{S_{z}, \delta} \int \frac{d^{3} q d^{3} Q}{(2 \pi)^{6}} \rho_{S_{z}}^{(\delta)}(\mathbf{q}, \mathbf{Q}) \\
& \times\left|\varphi_{\mathbf{q}, \mathbf{Q}}^{(\delta)}(\mathbf{r})\right|^{2}\left|\chi_{S_{z}}^{(\delta)}\left(\sigma_{1}, \sigma_{2}\right)\right|^{2},
\end{aligned}
$$

where passage to the thermodynamic limit is implied. Further, inserting Eq. (A7) into Eq. (58) and keeping in mind the relation

$$
\sum_{\sigma_{1}, \sigma_{2}}\left|\chi_{S_{z}}^{(\delta)}\left(\sigma_{1}, \sigma_{2}\right)\right|^{2}=1
$$

one can arrive at

$$
E_{\mathrm{int}} / V=\frac{1}{2} \int d^{3} r V(r) \sum_{S_{z}, \delta} \int \frac{d^{3} q d^{3} Q}{(2 \pi)^{6}} \rho_{S_{z}}^{(\delta)}(\mathbf{q}, \mathbf{Q})\left|\varphi_{\mathbf{q}, \mathbf{Q}}^{(\delta)}(\mathbf{r})\right|^{2} .
$$

Working in the dilution limit $n_{\ell / 2}, n_{\ell / 2-\ell^{\prime}} \rightarrow 0$, one can employ the low-momentum approximation of Eq. (62). This makes it possible to rewrite $E_{\text {int }}$ for $n_{\ell / 2}, n_{\ell / 2-\ell^{\prime}} \rightarrow 0$ in the form

$$
E_{\mathrm{int}} / V \simeq \frac{\eta_{\ell-\ell^{\prime}}^{(2)}}{2} \int d^{3} r V(r)\left|\varphi^{(2)}(\mathbf{r})\right|^{2},
$$

where

$$
\eta_{S_{z}}^{(\delta)}=\int \frac{d^{3} q d^{3} Q}{(2 \pi)^{6}} \rho_{S_{z}}^{(\delta)}(\mathbf{q}, \mathbf{Q})
$$




$$
\varphi^{(\delta)}(\mathbf{r})=\lim _{\mathrm{q}, \mathbf{Q} \rightarrow 0} \varphi_{\mathbf{q}, \mathbf{Q}}^{(\delta)}(\mathbf{r})
$$

Only the terms corresponding to $\delta=2$ make a contribution to the interaction energy (A9) in the dilution limit because $\varphi^{(1)}(\mathbf{r})$ is exactly equal to zero due to Eqs. (A4) and (A11). Following the arguments of Sec. IV one can replace Eq. (A9) by

$$
E_{\mathrm{int}} / V \simeq \eta_{\ell-\ell^{\prime}}^{(2)} \int d^{3} r V(r) \varphi^{2}(r)
$$

with $\varphi(r)$ obeying Eq. (11). Now, to get the final result, it only remains to have an idea about $\eta_{\ell-\ell^{\prime}}^{(2)}$. Equations (A7) and (69) lead to

$$
\sum_{S_{z}, \delta} \int \frac{d^{3} q d^{3} Q}{(2 \pi)^{6}} \rho_{S_{z}}^{(\delta)}(\mathbf{q}, \mathbf{Q})\left|\chi_{S_{z}}^{(\delta)}\left(\sigma_{1}, \sigma_{2}\right)\right|^{2}=n_{\sigma_{1}} n_{\sigma_{2}},
$$

provided that the normalization condition $(1 / V) \int_{V} d^{3} r \mid \varphi_{\mathbf{q}, \mathbf{Q}}^{(\delta)}$ $\times\left.(\mathbf{r})\right|^{2}=1$ is taken into account [see Eqs. (45) and (A1)]. From Eqs. (A2) and (A13) it is seen that

$$
\frac{1}{2} \int \frac{d^{3} q d^{3} Q}{(2 \pi)^{6}}\left[\rho_{\ell-\ell^{\prime}}^{(1)}(\mathbf{q}, \mathbf{Q})+\rho_{\ell-\ell^{\prime}}^{(2)}(\mathbf{q}, \mathbf{Q})\right]=n_{\ell / 2} n_{\ell / 2-\ell^{\prime}}
$$

It is natural to expect that $\rho_{\ell-\ell^{\prime}}^{(1)}(\mathbf{q}, \mathbf{Q})=\rho_{\ell-\ell^{\prime}}^{(2)}(\mathbf{q}, \mathbf{Q})$. Then, from Eqs. (A10) and (A14) we can find

$$
\eta_{\ell-\ell^{\prime}}^{(\delta)}=n_{\ell / 2} n_{\ell / 2-\ell^{\prime}} \text {. }
$$

Thus, for $n_{\ell / 2}, n_{\ell / 2-\ell^{\prime}} \rightarrow 0$ one gets

$$
E_{\text {int }} / V \simeq\left[4 \pi \hbar^{2}(a-b) / m\right] n_{\ell / 2} n_{\ell / 2-\ell^{\prime}}
$$

and, hence,

$$
\begin{gathered}
E / V \simeq\left(4 \pi \hbar^{2} a / m\right) n_{\ell / 2} n_{\ell / 2-\ell^{\prime}}, \\
E_{\mathrm{kin}} / V \simeq\left(4 \pi \hbar^{2} b / m\right) n_{\ell / 2} n_{\ell / 2-\ell^{\prime}} .
\end{gathered}
$$

At $n_{\ell / 2}=n_{\ell / 2-\ell^{\prime}}=n / 2$ Eqs. (A16)-(A18) reproduce the results of Sec. II taken in the leading order in $k_{F} a$. As was first pointed out in Ref. [15] and follows also from the considerations of Sec. IV and this appendix, trapping of fermions with two different $z$ projections of the single-particle spin allows one to operate with the system, where the effects of the pairwise interaction are more significant with respect to the situation of one $\operatorname{spin} z$ projection. This simplifies experimental searches for the BSC phase transition (see, for example, Ref. [14]).
[1] M. H. Anderson, J. R. Ensher, M. R. Matthews, C. E. Wieman, and E. A. Cornell, Science 269, 198 (1995).

[2] K. B. Davis et al., Phys. Rev. Lett. 75, 3969 (1995).

[3] C. C. Bradley, C. A. Sackett, J. J. Tollett, and R. G. Hulet, Phys. Rev. Lett. 75, 1687 (1995).

[4] E. P. Gross, Nuovo Cimento 20, 454 (1961); L. P. Pitaevskii, Zh. Eksp. Teor. Fiz. 40, 646 (1961) [Sov. Phys. JETP 13, 451 (1961)].

[5] L. Hau et al., Phys. Rev. A 58, R54 (1998).

[6] E. H. Lieb, R. Seiringer, and J. Yngvason, Phys. Rev. A 61, 043602 (2000).

[7] M. R. Andrews et al., Science 275, 637 (1997).

[8] S. Inouye et al., Phys. Rev. Lett. 87, 080402 (2001); F. Chevy et al., Phys. Rev. A 64, 031601 (2001).

[9] M. Naraschewski, H. Wallis, A. Schenzle, J. I. Cirac, and P. Zoller, Phys. Rev. A 54, 2185 (1996).

[10] F. Dalfovo, S. Giorgini, L. P. Pitaevskii, and S. Stringari, Rev. Mod. Phys. 71, 463 (1999).

[11] A. J. Legett, Rev. Mod. Phys. 73, 307 (2001).

[12] B. De Marco and D. S. Jin, Science 285, 1703 (1999).

[13] M. Greiner, C. A. Regal, and D. S. Jin, Nature (London) 426, 537 (2003).

[14] C. A. Regal, M. Greiner, and D. S. Jin, Phys. Rev. Lett. 92, 040403 (2002).

[15] H. T. C. Stoof et al., Phys. Rev. Lett. 76, 10 (1996).

[16] G. Roati, F. Riboli, G. Modugno, and M. Inguscio, Phys. Rev. Lett. 89, 150403 (2002).

[17] K. M. O'Hara, S. L. Hemmer, M. E. Gehm, S. R. Granade, and
J. E. Thomas, Science 298, 2179 (2002).

[18] L. D. Landau and E. M. Lifshitz, Statistical Physics (Pergamon Press, Oxford, 1980).

[19] A. Yu. Cherny and A. A. Shanenko, Eur. Phys. J. B 19, 555 (2001).

[20] A. Yu. Cherny and A. A. Shanenko, Phys. Rev. E 62, 1646 (2000); 64, 027105 (2001); Phys. Lett. A 292, 287 (2002).

[21] L. Vichi and S. Stringary, Phys. Rev. A 60, 4734 (1999).

[22] W. C. Stwalley, Phys. Rev. Lett. 37, 1628 (1976).

[23] E. Tiesinga, B. J. Verhaar, and H. T. C. Stoof, Phys. Rev. A 47, 4114 (1993).

[24] B. Militzer and E. L. Pollock, Phys. Rev. Lett. 89, 280401 (2002).

[25] K. Huang and C. N. Yang, Phys. Rev. 105, 767 (1957).

[26] H. A. Bethe and J. Goldstone, Proc. R. Soc. London, Ser. A 238, 551 (1957).

[27] V. Galitskii, Sov. Phys. JETP 7, 104 (1958).

[28] A. L. Fetter and J. D. Walecka, Quantum Theory of ManyParticle Systems (McGraw-Hill, New York, 1971).

[29] Our results are also valid in the situation of the pairwise potential combining the hard-sphere core with an attractive "tail" provided that the in-meduim bound states are "out of play." For example, this is a rather cold dilute Fermi gas that is still above the point of the BCS-like phase transition.

[30] All the derived results can easily be generalized to the case of another spin choice; see the Appendix.

[31] C. Kittel, Quantum Theory of Solids (Wiley, New York, 1963).

[32] T. D. Lee and C. N. Yang, Phys. Rev. 105, 1119 (1957). 
[33] K. A. Brueckner and K. Sawada, Phys. Rev. 106, 1117 (1957).

[34] E. H. Lieb and J. Yngvason, Phys. Rev. Lett. 80, 2504 (1998).

[35] D. S. Petrov et al., Phys. Rev. Lett. 84, 2551 (2000).

[36] E. Tiesinga et al., J. Res. Natl. Inst. Stand. Technol. 101, 505 (1996).

[37] Handbook of Mathematical Functions, edited by M. Abramowitz and I. Stegun (Dover, New York, 1970).

[38] N. N. Bogoliubov, Lectures on Quantum Statistics (Gordon and Breach, New York, 1970), Vol. 2.

[39] Strictly speaking, the total system momentum is nearly conserved if the volume is large enough. It exactly becomes a conserved quantity only in the thermodynamic limit.

[40] A. Yu. Cherny and A. A. Shanenko, Phys. Rev. B 60, 1276 (1999).

[41] Here we do not treat neglect of the bound pair states and the thermodynamic limit as approximations. 